\title{
Elevation of the Chemokine Pair CXCL10/ CXCR3 Initiates Sequential Glial Activation and Crosstalk During the Development of Bimodal Inflammatory Pain after Spinal Cord Ischemia Reperfusion
}

\author{
Qian Yu Da-Li Tian Ye Tian Xi-Tong Zhao Xue-Ying Yang \\ Department of Thoracic Surgery, The Fourth Affiliated Hospital of China Medical University, Shenyang, \\ China
}

\author{
Key Words \\ Astrocyte • CXCL10/CXCR3 • Inflammatory pain • Ischemia-reperfusion injury • Microglia
}

\begin{abstract}
Background/Aims: Spinal microglia and astrocytes are the main responders to the inflammatory cascade and process pain through various neural interactions. CXCL10 is a latephase protein that accelerates arteriogenesis during reperfusion through CXCR3. However, the early-phase expression (within $72 \mathrm{~h}$ postoperatively) of CXCL10 and CXCR3 during the development of ischemia-reperfusion (IR)-induced inflammatory pain remains unclear. We investigated whether this chemokine pair participates in glial interactions during early-phase IR injury. Methods: A rat model was induced by an 8-min occlusion of the aortic arch. Temporal assessments of mechanical and thermal allodynia and the protein levels of CXCL10 and CXCR3 were determined through measurements of paw withdrawal thresholds (PWTs) and paw withdrawal latencies (PWLs) and Western blotting assays. The co-localization of various cells with glial cells was detected by double immunofluorescence. The effects of CXCL10/CXCR3 on glial interactions were explored by intrathecal treatment with specific inhibitors (AMD487, minocycline and fluorocitrate) and recombinant CXCL10, and subsequent release of cytokines was assessed by ELISAs. Results: The IR injury initiated bimodal allodynia within $72 \mathrm{~h}$ of reperfusion, as illustrated by two W-shape trends in the PWTs and PWLs with two minima at 12 and $48 \mathrm{~h}$ post-IR. Allodynia was highly correlated with overexpression of CXCL10 and CXCR3, which were expressed in microglia at the early stage and in both microglia and astrocytes at the late stage, as shown by increased CXCL10 and CXCR3 immunoreactivities and doublelabeled cells. AMD487 and minocycline injections exerted comparable inhibitory effects on CXCR3 and Iba- 1 and on GFAP immunoreactivity at 12 and $48 \mathrm{~h}$ post-IR, and these inhibitory effects were only observed at $48 \mathrm{~h}$ following fluorocitrate injection. The levels of TNF- $\alpha$ and IL-6 showed variations in concert with the changes in Iba-1 and GFAP immunoreactivities.




\section{Cellular Physiology Cell Physiol Biochem 2018;49:2214-2228

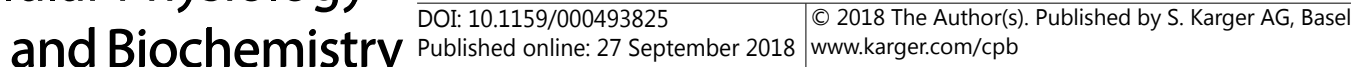 \\ Yu et al.: Sequential Glial Activation via CXCL10/CXCR3 Pair}

Recombinant CXCL10 injection reversed the abovementioned effects. Conclusion: The results showed that CXCL10/CXCR3 are involved in bimodal inflammatory pain during early-phase IR injury. The sequential activation of and crosstalk between microglia and astrocytes mediated through CXCR3 upregulation suggested that treatments targeting specific cell types are important in post-IR allodynia.

(C) 2018 The Author(s)

Published by S. Karger AG, Basel

\section{Introduction}

Spinal cord ischemia-reperfusion (IR) injury is considered the greatest clinical challenge that can occur during thoracoabdominal aortic aneurysm surgery. It usually inflicts severe and persistent IR-induced pain, which is characterized by symptoms of mechanical and cold allodynia but not involve direct nerve injury $[1,2]$. The pathophysiological mechanisms are multifactorial and have not been completely elucidated. Pain is processed by neural networks, and increasing lines of evidence suggest that IR-induced secondary inflammatory pain occurs concomitantly with glial activation both at remote sites and in an injured spinal cord through various forms of communication between neurons-glia or glia-glial cells [3, 4]. Microglia and astrocytes, which are the main glial cells in the spinal cord, constitute the blood-spinal cord barrier (BSCB) and act as the first responders to pathological stimuli [3, 5]. After activation, microglia transform to exhibit a macrophage-like morphology and drive rapid inflammatory amplification by triggering the transduction of nuclear factor (NF)- $\kappa B$ and the subsequent release of proinflammatory cytokines $[1,6]$. In rat models, astrocytes coordinate with microglia to exacerbate inflammation throughout the spinal cord and simultaneously release cytokines that ultimately decrease the pain threshold [3-5, 7]. Our results demonstrate that glial cells are activated in a closely interdependent manner and form a functional unit for the development and maintenance of inflammatory pain after spinal cord IR injury. Thus, treatments that regulate these links might represent novel therapeutic targets.

The sequential activation of and crosstalk between microglia and astrocytes is now known to be performed by soluble mediators, such as adenosine, growth factors, inflammatory chemokines and cytokines $[3,8,9]$. Among these mediators, chemokines are 8-14-kDa secreted proteins with conserved cysteine residues in their sequences. The chemokine C-X-C motif ligand (CXCL)10, which is also known as interferon (IFN)- $\gamma$-inducible protein 10 (IP-10), is a pleiotropic chemokine that belongs to the C-X-C subfamily [10]. As the name implies, this chemokine is secreted by several cell types (e.g., leukocytes, macrophages and endothelial cells) in response to IFN- $\gamma$ and recruits these cells to sites of infection or inflammation after binding to C-X-C motif receptor (CXCR)3 [10, 11]. The chemokinereceptor pair CXCL10/CXCR3 is widely expressed throughout the central nervous system (CNS) and produces different responses in CNS pathology depending on its expression in different cell types [11-13]. In addition to its "classical" role in the chemotaxis of responsive cells, accumulating lines of evidence highlight newfound effects on modulating glial interactions during the induction of pain perception [12, 13]. CXCL10 is expressed at low levels in the spinal cord under normal conditions, but upon stimulation, the expression of this chemokine is substantially increased at sites where glial cells accumulate [11]. In vivo immunofluorescence staining has also shown that CXCR3 co-localizes with spinal microglia and astrocytes in a rat model of bone cancer-induced pain $[11,14]$. The neutralization of CXCR3 significantly reduces the expression of markers of activated microglia and astrocytes as well as the levels of proinflammatory cytokines and chemokines, such as CCL2 and CXCL10 $[11,12,14]$. Thus, microglia and astrocytes participate in the development of inflammatory pain and act as the targets of the chemokine-receptor pair CXCL10/CXCR3. Notably, CXCL10 has been previously described as a late-phase protein involved in accelerating arteriogenesis during the perfusion phase, and CXCL10 expression is significantly increased on day 4 after hindlimb ischemia [15]. However, little is known about the early-stage expression of CXCL10 after spinal cord IR injury (within 3 days post-surgery) and whether the chemokine-receptor 
pair CXCL10/CXCR3 participates in spinal glial interactions in IR-induced inflammatory pain. Thus, in this study, we first characterize the early changes in CXCL10 and CXCR3 expression in major spinal glial cells in a rat model of IR-induced pain. We then explore the roles of the chemokine-receptor pair CXCL10/CXCR3 in IR-induced inflammatory pain by administering intrathecal injections of a specific CXCR3 antagonist, glial inhibitors and recombinant rat CXCL10 protein (rCXCL10). Here, we provide the first evidence demonstrating the involvement of CXCL10/CXCR3 in the development of bimodal inflammatory pain during the early reperfusion phase. These factors act by regulating the interactions between microglia and astrocyte, and thus, treatments targeting specific cell types are of great importance.

\section{Materials and Methods}

\section{Animals}

SD rats weighting 180-220 g were obtained from the Animal Center of China Medical University (Shenyang, China). All the rats were neurologically normal and were placed in separate cages at least 7 days before surgery. The animals had free access to food and water and were maintained at $22-24^{\circ} \mathrm{C}$ with a 12-h light/dark cycle. All experimental protocols were approved by the Ethics Committee of China Medical University. All invasive procedures were performed under anesthesia to minimize suffering in accordance with the Guide for the Care and Use of Laboratory Animals (U.S. National Institutes of Health Publication No. 85-23, National Academy Press, Washington DC, revised 1996).

\section{Rat model of IR-induced pain}

IR-induced pain was established in the rats through an 8-min occlusion of the aortic arch [1]. Briefly, all the rats were anesthetized with $2 \%$ sevoflurane and placed in a lateral position to expose the aortic arch through a cervicothoracic approach. The non-invasive artery clip was first clamped between the left common carotid artery and the left subclavian artery for $8 \mathrm{~min}$ and then removed to imitate IR. Ischemia was confirmed through a $90 \%$ decrease in blood flow at the tail artery, and reperfusion was detected through the restoration of flow on a Doppler monitor (Moor Instruments, Axminster, Devon, UK). Sham-operated rats underwent the same procedure without clamping. All the rats were observed for $72 \mathrm{~h}$ after reperfusion.

\section{Experimental protocol}

Part I. IR-induced pain was analyzed as mentioned above. Forty-two rats were randomly assigned to the sham group or the IR group to investigate sensory changes and the time course of CXCL10 and CXCR3 expression at 12-h intervals during the 72-h period after reperfusion.

Part II. Four other groups were established to explore the roles of the chemokine-receptor pair CXCL10/ CXCR3 in IR-induced pain. One hundred forty-four rats were intrathecally injected with 10- $\mu$ l volumes of normal saline, AMG487 (CXCR3 inhibitor, 20 g, Sigma-Aldrich, MO, USA), minocycline (microglial inhibitor, $10 \mathrm{nmol} / \mu \mathrm{l}$, Nichiiko, Toyama, Japan), fluorocitrate (astrocytic inhibitor, $1 \mathrm{nmol} / \mu \mathrm{l}$, Sigma-Aldrich, MO, USA), of rCXCL10 (1.0 $\mu \mathrm{g} / \mu \mathrm{l}$, Novus Biologicals, CO, USA). The correct placement of the intrathecal injection into the L5-6 segments of the intervertebral space was confirmed by tail flicking. The rats were euthanized at 12 and $48 \mathrm{~h}$ with an overdose of sevoflurane. The L4-6 segments of the spinal cords were rapidly collected for further analyses.

\section{Sensory behavioral assessments}

Mechanical allodynia was measured by recording the PWTs using von Frey filaments (37450, Ugo Basile, Dublin, Ireland), as previously described [1]. The tests were performed by another observer who was blinded to the experimental protocols. One of the hind-limb paws was stimulated with a series of filaments with logarithmically ascending forces of $2,4,6,8,10$, and $15 \mathrm{~g}$. The PWT was recorded as the average value of the five minimum levels of forces that prompted the rat to withdraw its paw. Each stimulus was maintained for $3 \mathrm{~s}$ and applied at 15 -s intervals.

Thermal allodynia was measured 15 min after the PWT measurements by recording the PWL using a hot plate (35150, Ugo Basile, Dublin, Ireland) [1]. The PWL was determined as the average from triplicate measurements of the time that the rat stayed on a hot plate at $50^{\circ} \mathrm{C}$ before it initially clearly withdrew 


\section{Cellular Physiology Cell Physiol Biochem 2018;49:2214-2228

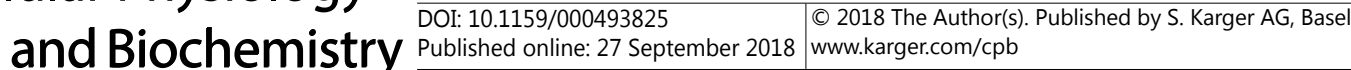 \\ Yu et al:: Sequential Glial Activation via CXCL10/CXCR3 Pair}

its paws. The interval was $10 \mathrm{~min}$, and each exposure time was limited to $25 \mathrm{~s}$ to avoid permanent tissue damage. Allodynia was defined by statistically significant decreases in the PWLs and PWTs compared with the baseline values.

\section{Western blots}

Spinal cord tissues were collected from each rat and purified using a protein extraction kit according to the manufacturer's instructions (KC-415, KangChen, Shanghai, China). Protein concentrations were quantified using the Bradford method. Proteins were separated by electrophoresis on $8 \%$ polyacrylamide gels and then transferred to polyvinylidene fluoride membranes. The membranes were incubated overnight at $4^{\circ} \mathrm{C}$ with primary antibodies against Iba-1 (1:200, Wako, Osaka, Japan), GFAP (1:200, Abcam, Cambridge, MA, USA), CXCL10 (1:100, Abcam, Cambridge, MA, USA) and CXCR3 (1:100, Abcam, Cambridge, MA, USA) and then with horseradish peroxidase-labeled secondary antibodies $(1: 1,000$, Beyotime Biotechnology, Shanghai, China) at room temperature for $60 \mathrm{~min}$. The bands were visualized using an ECL Plus Kit (Beyotime Biotechnology, Shanghai, China), and the integrated densities were quantified using Quantity One software (Bio-Rad Laboratories, Milan, Italy).

\section{Double immunofluorescence staining}

The cellular distributions of CXCL10 and CXCR3 were determined using the microglia marker Iba1 and the astrocyte marker GFAP, as previously described [1]. L4-6 spinal cords were post-fixed with $4 \%$ paraformaldehyde for $12 \mathrm{~h}$ and dehydrated with $20 \%$ sucrose for $24 \mathrm{~h}$ at $4^{\circ} \mathrm{C}$. The tissues were then frozen and sliced into 20- $\mu \mathrm{m}$ sections using a cryostat (Leica, Leica Biosystems, USA). The sections were then blocked in $10 \%$ bovine serum albumin and incubated with a primary rabbit polyclonal anti-CXCL10 antibody (1:100, PeproTech, NJ, USA), rabbit monoclonal anti-CXCR3 antibody (1:100, Abnova, Taiwan, China), mouse monoclonal anti-GFAP antibody (1:500, Abcam, USA), and mouse monoclonal anti-Iba-1 antibody $\left(1: 400\right.$, Abcam, USA) overnight at $4^{\circ} \mathrm{C}$. The sections were subsequently washed and incubated with Alexa Fluor 488-conjugated donkey anti-rabbit IgG (1:500, Cell Signaling Technology, MA, USA) and Alexa Fluor 594-conjugated donkey anti-mouse IgG (1:500, Cell Signaling Technology, USA) for $2 \mathrm{~h}$ at room temperature. Images of the immunofluorescence staining were obtained using a Leica confocal microscope (Leica Microsystems, Buffalo Grove, IL, USA) and analyzed using Image-Pro Plus software (Media Cybernetics, TX, USA).

\section{ELISAs for determining the TNF- $\alpha$ and IL-6 contents}

After the spinal cord samples were homogenized and centrifuged, the spinal supernatants were collected to determine the TNF- $\alpha$ and IL- 6 contents using ELISA kits (R\&D Systems, Minneapolis, MN, USA) according to the manufacturer's instructions. The concentration in each sample was calculated based on the standard curve and is expressed as $\mathrm{pg} / \mathrm{mg}$ protein.

\section{Statistical analysis}

All the data are presented as the means \pm standard errors of the means (SEMs) and were analyzed using SPSS software (version 17.0, SPSS Inc., Chicago, IL, USA). The results from the immunofluorescence staining, Western blotting and ELISAs were analyzed by one-way analysis of variance (ANOVA) followed by Tukey's post hoc test. The results of the behavioral assessments were compared by two-way repeated ANOVA followed by the Bonferroni post hoc test. A $P$ value $<0.05$ was considered significant.

\section{Results}

Bimodal mechanical and thermal allodynia developed in the IR-induced pain model

All the rats included in the experiments exhibited normal neurological function before IR. Compared with the baseline measurements, the ipsilateral paw withdrawal thresholds (PWTs) and paw withdrawal latencies (PWLs) were noticeably lower throughout the entire observation period, indicating that IR promoted the development and maintenance of mechanical and thermal allodynia (Fig. 1, $P<0.05$ ). Moreover, the time course of PWTs and PWLs appeared to exhibit a W-shaped curve, with the two lowest points at $12 \mathrm{~h}$ and $48 \mathrm{~h}$. In 
addition, the values of PWTs and PWLs measured at $48 \mathrm{~h}$ and later time points were notably lower than the values measured at $12 \mathrm{~h}(P<0.05)$.

Time course of the expression of the CXCL10 and CXCR3 proteins in the IR-induced pain model

Chemokines are emerging as important regulators of glial activation during IR-induced pain $[3,12]$. As shown in Fig. 1C and D, the CXCL10 and CXCR3 proteins were expressed at low levels postoperatively in sham-operated rats, whereas in the rats the underwent IR, the levels of these proteins increased over time, starting at $12 \mathrm{~h}$ and remaining at a substantially high level at later time points $(P<0.05)$.

\section{Glial localization of the CXCL10 and CXCR3 proteins in the IR-induced pain model}

Because bimodal allodynia developed in the IR injury model and potentially involved glial cells, we next investigated whether the chemokine-receptor pair CXCL10/CXCR3 was expressed in spinal glial cells by performing double immunofluorescence at both 12 and $48 \mathrm{~h}$ post-IR. Representative micrographs showed increased immunoreactivities of ionized

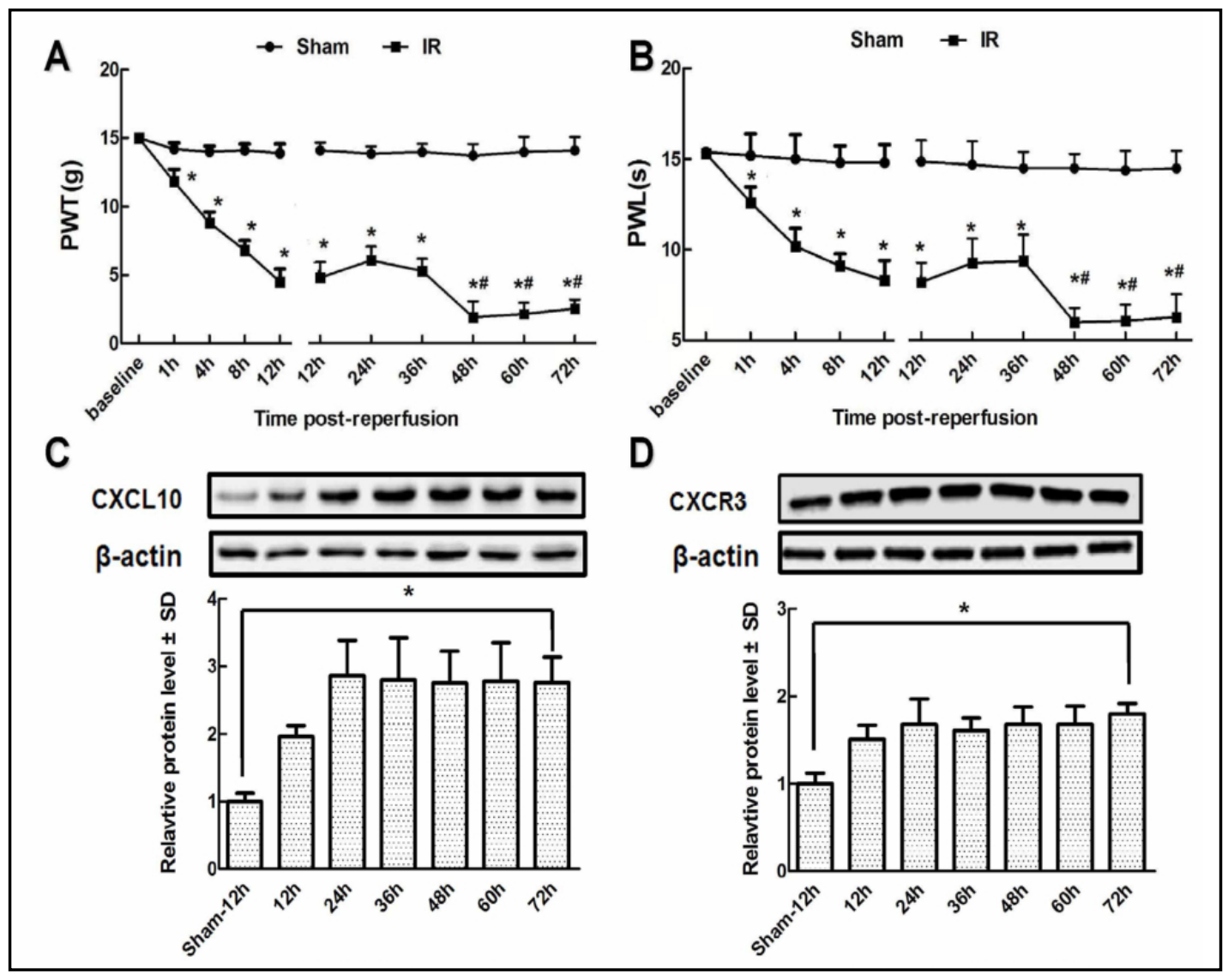

Fig. 1. Changes in mechanical and thermal allodynia and spinal expression of the chemokine-receptor pair CXCL10/CXCR3 in the IR-induced pain model. (A and B) The development of mechanical and thermal sensitivity in IR-induced pain was evaluated using von Frey filaments and a hot plate for $72 \mathrm{~h}$ after reperfusion. Compared with the sham-operated rats, significant decreases in PWTs and PWLs were observed throughout the observation period in the rats with IR injury. The curves exhibited a W-shape, with the two lowest points at 12 and $48 \mathrm{~h}$ post-IR, suggesting that IR evoked bimodal allodynia. (C and D) Representative blots for CXCL10 and CXCR3 were evaluated at 12-h intervals for $72 \mathrm{~h}$ after reperfusion. The relative protein levels were calculated as fold increases compared with the sham group. The data are reported as the means \pm SEMs. ${ }^{*} \mathrm{P}<0.05$ compared with the sham group, ${ }^{*} \mathrm{P}<0.05$ compared with the IR group. 
calcium-binding adaptor molecule- 1 (Iba-1) and glial fibrillary acidic protein (GFAP) in the rats that underwent IR but not in the sham-operated rats at these two time points (Fig. 2A), indicating that activated microglia and astrocytes participated in the development of IRinduced pain. Furthermore, most of the fluorescently labeled CXCL10 was co-localized with the Iba-1- and GFAP-positive cells (indicated by arrows) in the same samples. In addition, quantification of the immunoreactivities and the number of double-labeled cells confirmed that CXCL10 expression was indeed increased in activated microglia and astrocytes (Fig. 2B and $\mathrm{C}, P<0.05$ ).

Slight differences in the distribution of CXCL10 were detected, and strong CXCR3 immunoreactivity was observed only in Iba-1-positive cells and not in GFAP-positive calls at $12 \mathrm{~h}$ post-IR, whereas at $48 \mathrm{~h}$ post-IR, CXCR3 immunoreactivity was increased in both Iba1- and GFAP-labeled cells (Fig. 3A). According to the results of the quantitative analysis, the increased level of CXCL10 might increase the binding of microglia to CXCR3 during the early stage of bimodal allodynia, whereas the later stage involved both microglia and astrocytes (Fig. 3B and $\mathrm{C}, P<0.05$ ).

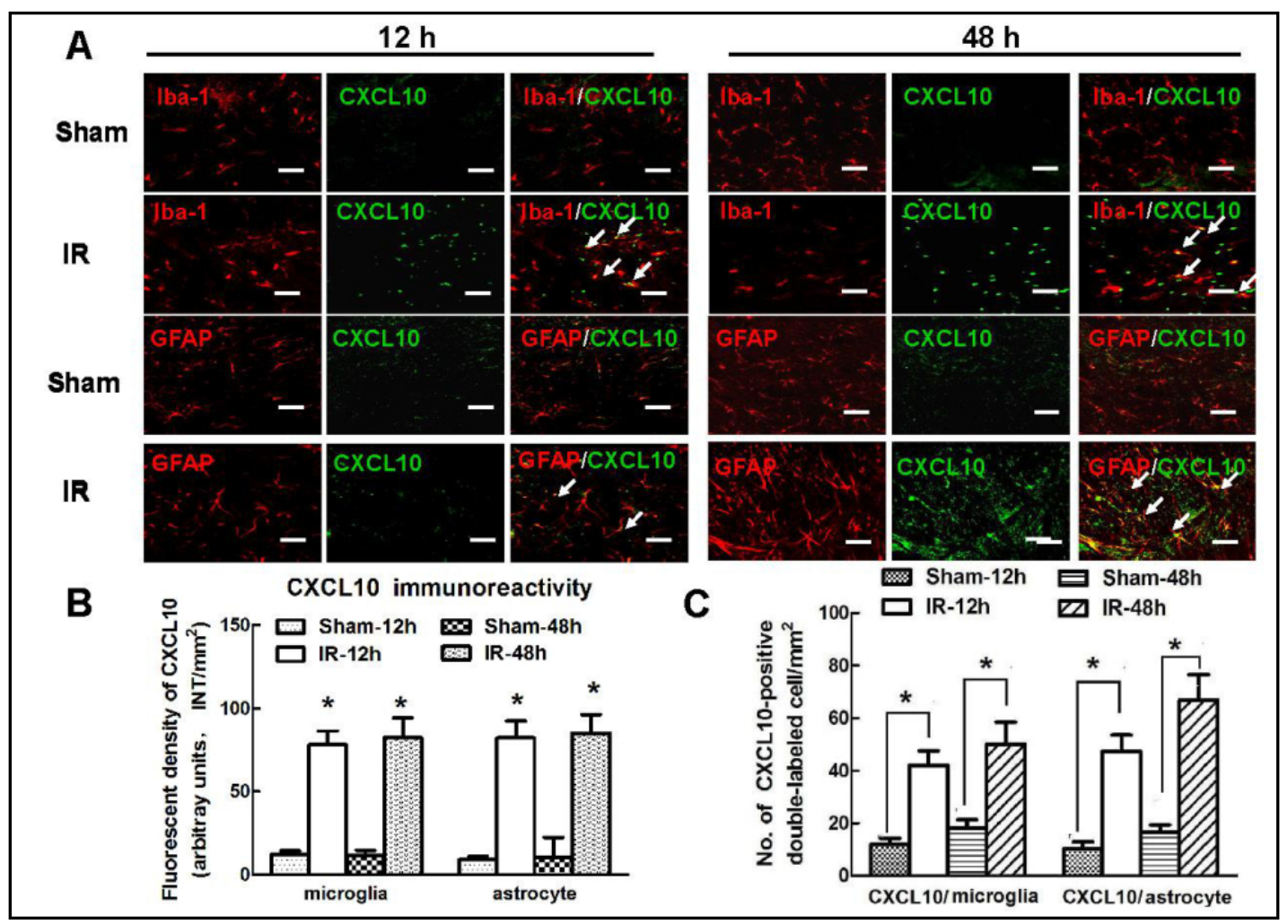

Fig. 2. Glial localization of CXCL10 expression in the IR-induced pain model. (A) Representative micrographs showing the co-localization of microglia (Iba-1, red) and astrocytes (GFAP, red) expressing the chemokine CXCL10 (green) at 12 and $48 \mathrm{~h}$ post-IR. Co-localization is shown by a yellow signal. The scale bars represent $100 \mu \mathrm{m}$. (B) Quantification of the fluorescence intensity of CXCL10 in the spinal cord. (C) Quantification of the number of double-labeled CXCL10-positive cells in the spinal cord. IR activated microglia and astrocytes via the overexpression of CXCL10 at 12 and $48 \mathrm{~h}$ post-IR. The data represent the averages of three independent samples and are reported as the means \pm SEMs. ${ }^{*} \mathrm{P}<0.05$ compared with the sham group. 


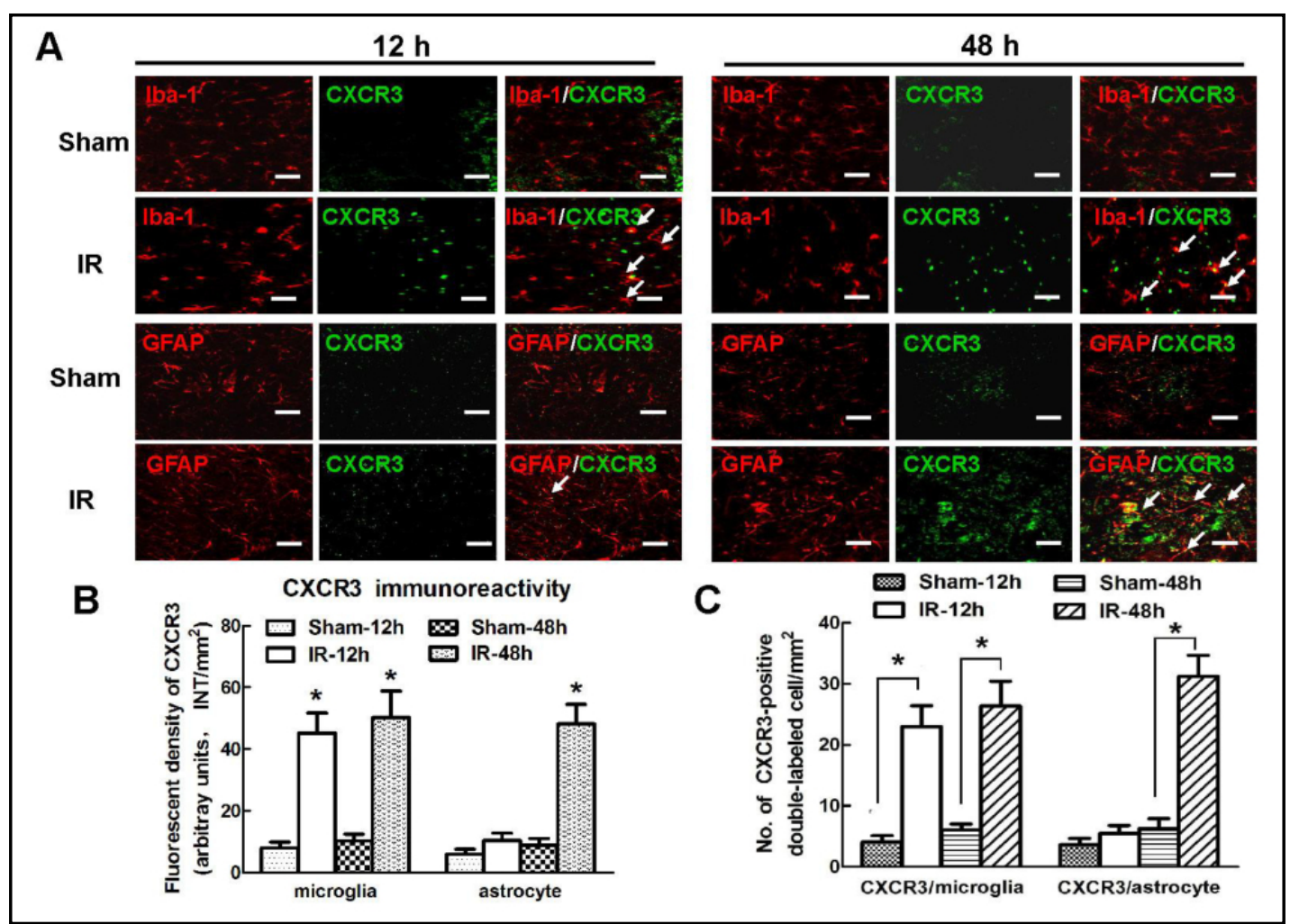

Fig. 3. Glial localization of CXCR3 expression in the IR-induced pain model. (A) Representative micrographs showing the co-localization of microglia (Iba-1, red) and astrocytes (GFAP, red) with the chemokine receptor CXCR3 (green) at 12 and $48 \mathrm{~h}$ post-IR. Co-localization is shown as a yellow signal. The scale bars represent $100 \mu \mathrm{m}$. (B) Quantification of the fluorescence intensity of CXCR3 in the spinal cord. (C) Quantification of the number of double-labeled CXCR3-positive cells in the spinal cord. IR activated microglia through the overexpression of CXCR3 at both 12 and $48 \mathrm{~h}$ post-IR, but activated astrocytes exhibited only CXCR3 overexpression at $48 \mathrm{~h}$ post-IR. The data are presented as averages of three independent samples and are reported as the means \pm SEMs. $* \mathrm{P}<0.05$ compared with the sham group.

Roles of the CXCL10/CXCR3 pair in glial activation and crosstalk in the IR-induced pain model

Based on the results described above, glial activation depended on the overexpression of CXCR3. Further interactions between these cells and specific cellular targets were explored by administering intrathecal injections of AMG487 (a CXCR3 inhibitor), minocycline (a microglia inhibitor), fluorocitrate (an astrocyte inhibitor) and rCXCL10. Representative blots and quantitative analyses showed significantly higher levels of the CXCR3 protein in all IRoperated rats than in sham-operated rats at both 12 and $48 \mathrm{~h}$ post-IR (Fig. $4 \mathrm{~A}$ and $\mathrm{B}, P<0.05$ ). In addition, these increases were specifically antagonized by the intrathecal injection of AMG487 and synergistically aggravated by rCXCL10 $(P<0.05)$.

Because CXCR3 expression was closely related to glial activation, we further evaluated the statuses of microglia and astrocytes via immunofluorescence staining because this method intuitively displays the extent of activation through different fluorescence intensities [1]. Microglia, which are immunoreactive for Iba-1, were highly activated and showed increased fluorescence intensities at $12 \mathrm{~h}$ and $48 \mathrm{~h}$ post-IR. These values were equivalently decreased by the injection of AMD487 and minocycline (Fig. 4C and D, $P<0.05$ ). Similarly, astrocytes were gradually activated over time, resulting in a slight increase in the fluorescence intensity of GFAP at $12 \mathrm{~h}$ but a notable increase at $48 \mathrm{~h}(P<0.05)$. In addition, the intrathecal injection of AMD487 and fluorocitrate reversed the above changes $(P<0.05)$, whereas the injection of 


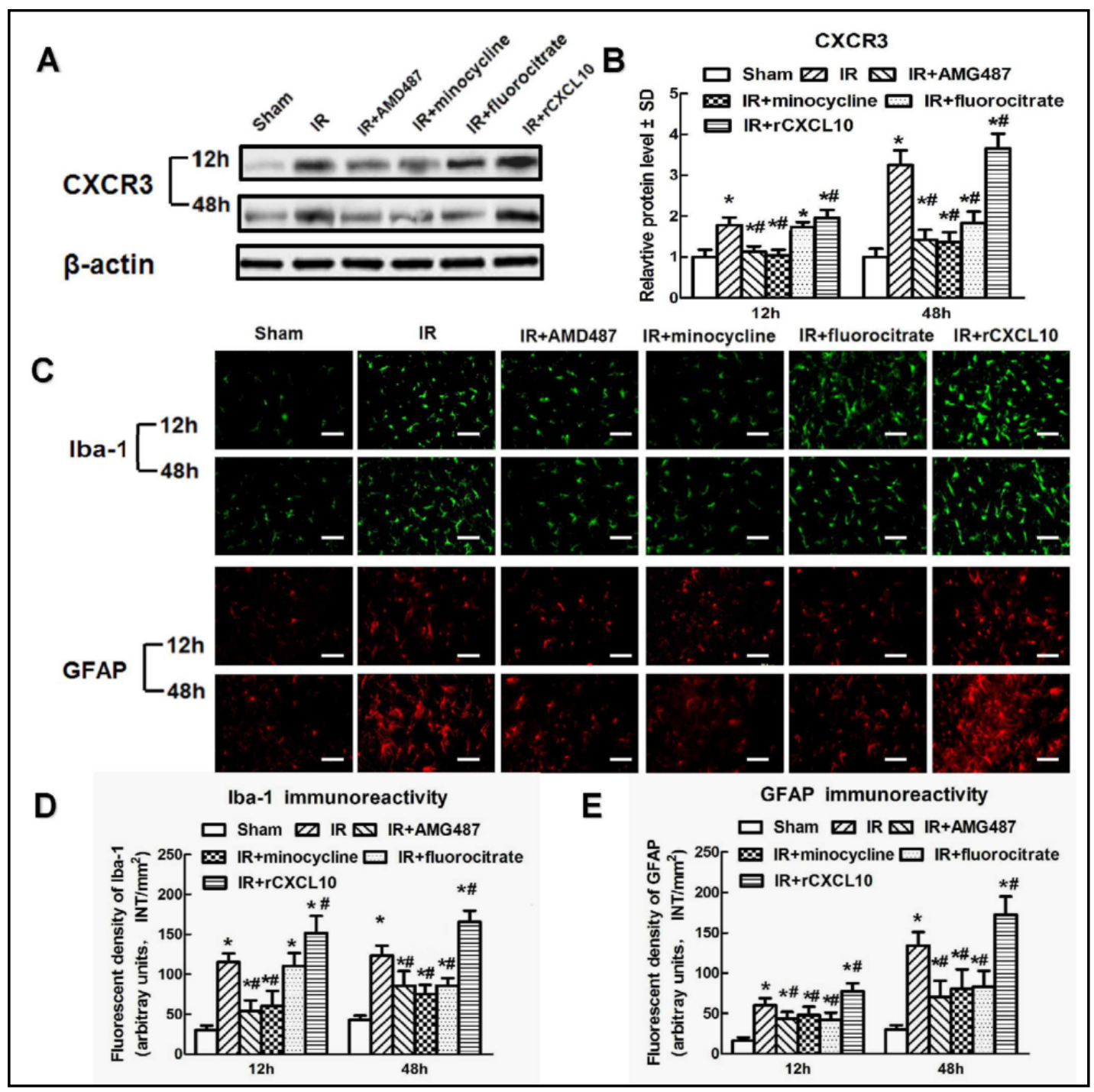

Fig. 4. Roles of the CXCL10/CXCR3 pair in sequential microglia and astrocyte activation and crosstalk in the IR-induced pain model. These roles were explored using agents that act on specific cellular targets. (A) Representative blots showing the CXCR3 levels in animals that received intrathecal injections of AMG487 (CXCR3 inhibitor), minocycline (microglia inhibitor), fluorocitrate (astrocyte inhibitor) or rCXCL10 at 12 and $48 \mathrm{~h}$ post-IR. (B) The levels of the CXCR3 protein were quantified as fold increases compared with the sham group. (C) The changes in microglia and astrocyte activation are intuitively displayed through immunofluorescence staining for Iba-1 and GFAP. The scale bars represent $100 \mu \mathrm{m}$. (D and E) Quantification of the fluorescence intensities of Iba- 1 and GFAP in the spinal cord. These data provide evidence for the sequential activation of and crosstalk between microglia and astrocytes through CXCR3 overexpression. The data are presented as the means \pm SEMs. ${ }^{*} \mathrm{P}<0.05$ compared with the sham group, ${ }^{\#} \mathrm{P}<0.05$ compared with the IR group.

rCXCL10 aggravated these changes $(P<0.05)$.

Additionally, the immunoreactivities of CXCR3, Iba-1 and GFAP were detected simultaneously by injecting minocycline or fluorocitrate to explore potential glial cell interactions. As shown in Fig. 4A, minocycline reduced the expression of CXCR3 to the same extent as the injection of AMD487 at 12 and $48 \mathrm{~h}$ post-IR $(P>0.05)$. In addition, the intrathecal injection of minocycline similarly prevented increases in the immunoreactivity of both Iba-1 and GFAP at 12 and $48 \mathrm{~h}$ post-IR (Fig. 4C, $P>0.05$ ). Similar to the AMD487 treatment, fluorocitrate decreased CXCR3 expression and GFAP and Iba-1 immunoreactivity 


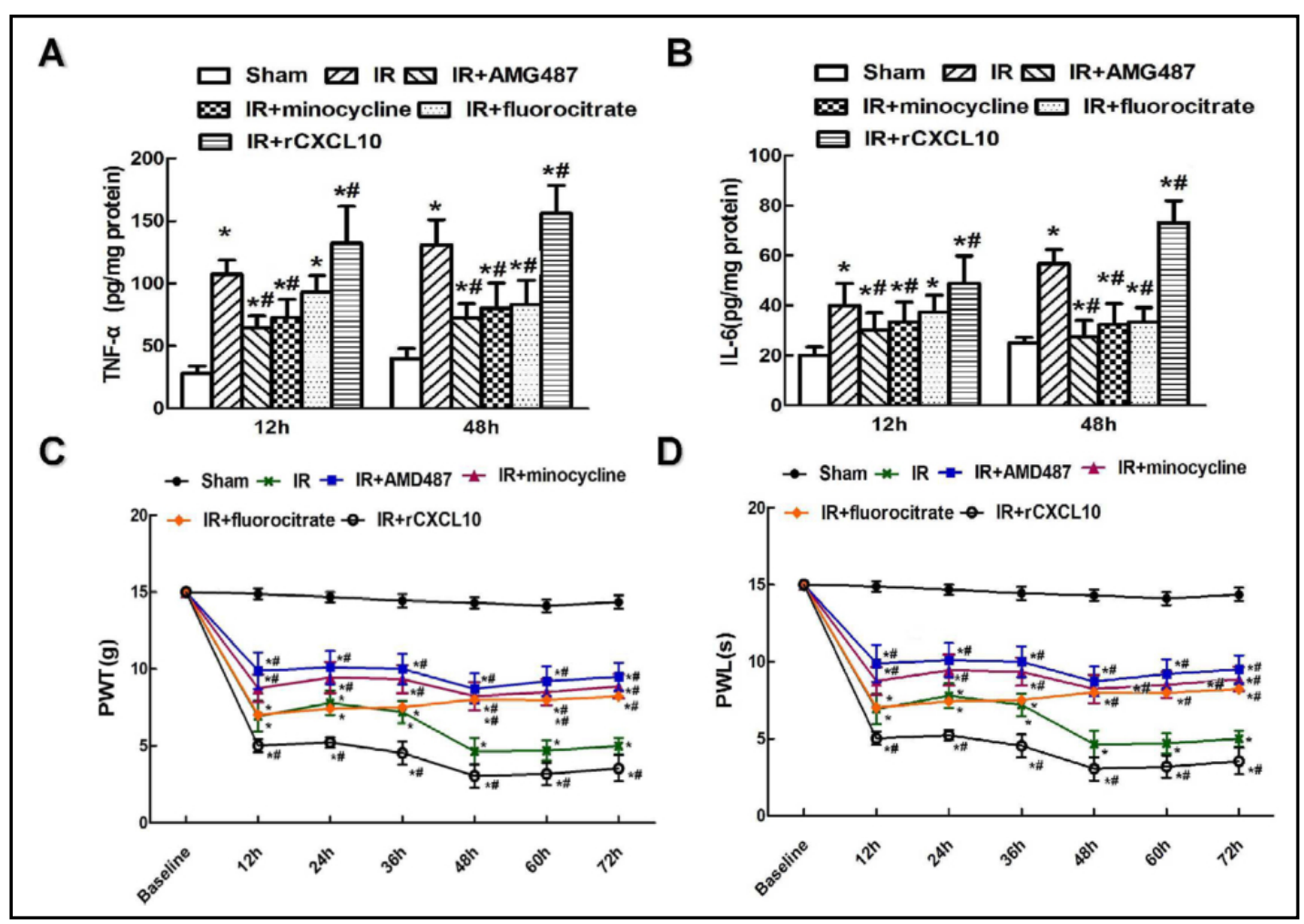

Fig. 5. Roles of the CXCL10/CXCR3 pair in the release of proinflammatory cytokines and the development of allodynia in the IR-induced pain model. These roles were explored using specific cellular targets. (A-B) The TNF- $\alpha$ and IL-6 levels in mice injected with AMG487, minocycline, fluorocitrate or rCXCL10 at 12 and $48 \mathrm{~h}$ post-IR were quantified by ELISAs. (C-D) The changes in the mechanical and thermal sensitivity of rats injected with AMG487, minocycline, fluorocitrate or rCXCL10 were evaluated by recording the PWTs and PWLs. CXCR3 overexpression was closely associated with microglia and astrocyte activation and the release of the proinflammatory cytokines TNF- $\alpha$ and IL-6, and these effects ultimately led to the development of allodynia. The data are presented as the means \pm SEM. ${ }^{*} \mathrm{P}<0.05$ compared with the sham group, ${ }^{*} \mathrm{P}<0.05$ compared with the IR group.

at $48 \mathrm{~h}$ post-IR (Fig. $4 \mathrm{~A}$ and $\mathrm{C}, P>0.05$ ). Significant differences in Iba-1 immunoreactivity were not observed between the IR and fluorocitrate groups at $12 \mathrm{~h}$ post-IR $(P>0.05)$.

Roles of the CXCL10/CXCR3 pair in the development of inflammatory allodynia in the IRinduced pain model

We measured the TNF- $\alpha$ and IL-6 contents through ELISAs to test whether the chemokine-receptor pair CXCL10/CXCR3 affects the release of proinflammatory cytokines from astrocytes and microglia. According to the results of the quantitative analysis, IR induced substantial increases in the TNF- $\alpha$ and IL- 6 levels at 12 and $48 \mathrm{~h}$ post-IR compared with the sham procedure (Fig. $5 \mathrm{~A}, P>0.05$ ). In addition, the minocycline injection produced similar decreases in the production of TNF- $\alpha$ and IL- 6 at 12 and $48 \mathrm{~h}$ post-IR as the AMD487 injection, whereas fluorocitrate exerted similar effects at $48 \mathrm{~h}$ post-IR $(P<0.05)$. In contrast, the injection of rCXCL10 increased the release of TNF- $\alpha$ and IL- 6 at these two time points $(P<0.05)$. No significant differences between the IR and fluorocitrate groups were observed at $12 \mathrm{~h}$ post-IR $(P>0.05)$.

As expected, the sensory behavioral assessments showed that the intrathecal injections of AMG487 and minocycline significantly increased the PWTs and PWLs compared with the IR group throughout the entire observation period, whereas the injection of fluorocitrate only increased the PWTs and PWLs at $48 \mathrm{~h}$ post-IR and later time points (Fig. $4 \mathrm{~A}, P<0.05$ ). In contrast, the intrathecal injection of rCXCL10 synergistically decreased the PWTs and 
PWLs at all observed time points (Fig. 4A, $P<0.05$ ). No significant differences were observed within $48 \mathrm{~h}$ post-IR between the rats that underwent IR and those that received fluorocitrate $(P>0.05)$.

\section{Discussion}

The chemokine-receptor pair CXCL10/CXCR3 is distributed in microglia and astrocytes, suggesting that these proteins play important physiological and/or pathological roles in regulating chemotaxis and behavioral hypersensitivity [11-12,14]. In the present study, we obtained the first evidence showing that the spinal expression of CXCL10/CXCR3 increases in a time-dependent manner during the early stage of reperfusion (within 3 days) and initiates bimodal inflammatory allodynia by regulating the sequential activation of and crosstalk between microglia and astrocytes and the release of the proinflammatory cytokines TNF- $\alpha$ and IL- 6 in a rat model of IR-induced pain.

Consistent with a previous study of IR injury, two phases of the inflammatory response were observed in a rat model during the early stage of reperfusion [6]. In the present study, we also observed bimodal allodynia during the early phase of reperfusion because $\mathrm{W}$-shaped trends in the PWTs and PWLs were detected within the first $72 \mathrm{~h}$ following reperfusion (Fig. 1). CXCL10 is a well-known proinflammatory chemokine that is expressed at a very low level under normal conditions $[11,16]$. Upon stimulation, however, the levels of CXCL10 increase rapidly, and this protein is necessary for the onset of the inflammatory cascade [16]. Thus, we further characterized the expression of the CXCL10 protein in the spinal cord by Western blotting. CXCL10 was expressed at different levels in rats with and without IR, indicating that CXCL10 might participate in the pathogenesis of IR-induced pain (Fig. 1). A recent study showed that CXCL10 is widely expressed at the sites of glial proliferation during the reperfusion phase, which suggests that it induces glial activation during pain processing [13]. We thus further explored the glial localization of CXCL10 in the spinal cord through double immunofluorescence staining. The enlarged area and strength of the immunostaining determined through the image analysis corresponded well with the sites showing activated microglia and astrocytes after injury [17, 18]. As previously described, spinal glial activation can be determined by measuring Iba-1 (microglia) and GFAP (astrocytes) immunoreactivity [14]. CXCL10 was upregulated in both microglia and astrocytes because the increased fluorescent labeling of CXCL10 coincided with increased Iba-1 and GFAP staining in the injured spinal cords (Fig. 2). In addition, consistent with the Western blotting results, notably stronger CXCL10 immunoreactivity and greater numbers of double-labeled cells were detected at $48 \mathrm{~h}$ post-IR than at $12 \mathrm{~h}$, confirming that CXCL10 participates in the development of bimodal allodynia by regulating the activated populations of microglia and astrocytes.

The chemokine receptor CXCR3, for which CXCL10 is a major ligand, is expressed on different cells in which its activation leads to different cellular activities [19, 20]. Activated immune cells express high levels of CXCR3 $[11,21]$. In addition to regulating chemotaxis and inflammatory responses, CXCR3 has been shown to mediate neuron-glia and/or gliaglia interactions in response to acute brain injury and ischemia [22-24]. Similarly, our study showed that the expression of the CXCR3 protein gradually increased in parallel with increases in CXCL10 expression (Fig. 1) but was differentially and sequentially upregulated in microglia and astrocytes at 12 and $48 \mathrm{~h}$ post-IR (Fig. 3). Some studies have reported different temporal profiles for the activation of microglia and astrocytes in the progression of CNS injury [22,23]. Two hours after reperfusion, the number and activation of microglia are increased in the spinal cord, and these effects are more pronounced at 24 and 48 $\mathrm{h}$ [25]. In astrocytes, no increased immunoreactivity of GFAP was visible until $12 \mathrm{~h}$ after reperfusion, and this increase became more intense at $24 \mathrm{~h}$ and later time points [25]. Moreover, in response to a lesion in the entorhinal cortex, microglia are activated in wildtype mice and migrate to the lesion zone within the first 3 days [22]. After a delay, astrocytes

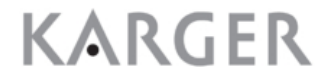


are activated by hypertrophy, and increased GFAP expression is observed 8 days after the injury [22]. Similarly, the double-immunofluorescence staining performed in the present study also showed an early increase in CXCR3 expression in activated microglia, and this increase began at $12 \mathrm{~h}$ post-IR and was intensified at $48 \mathrm{~h}$. In contrast, the increase in CXCR3 expression on astrocytes was significantly delayed and was only detected at $48 \mathrm{~h}$ post-IR. In combination with the evidence that CXCR3 expression affects the transformation of the functional properties of microglia after CNS injury [11, 22], this sequential activation of spinal microglia and astrocytes during IR-induced pain suggests that CXCR3 might provide the excitatory glial signal that spreads from the early activation of microglia to prompt the late activation of astrocytes. We performed parallel experiments by administering intrathecal injections of CXCR3-, microglia- and astrocyte-specific inhibitors and evaluated the changes in CXCR3 expression and the immunoreactivity of Iba-1 and GFAP simultaneously within the same region to confirm this hypothesis. Because the microglia inhibitor minocycline and the CXCR3 inhibitor AMD487 exhibited similar effects in that they prevented increases in CXCR3 expression and Iba-1 immunoreactivity at both 12 and 48 h post-IR, we postulated that activated microglia might be one of the initial sources of CXCR3. Moreover, the injection of AMD487 and the astrocyte inhibitor fluorocitrate decreased CXCR3 expression and GFAP immunoreactivity to similar extents at $48 \mathrm{~h}$ post-IR, confirming that the delayed activation of astrocytes was also mediated by the CXCR3 receptor. This result is consistent with previous findings showing that CXCR3 neutralization significantly reduces the levels of markers of activated microglia and astrocytes in models of cancer pain and demyelination diseases $[11,14]$. Consistent with these findings, amplifying the function of CXCR3 with recombinant CXCL10 evoked greater activation of microglia and astrocytes at these two time points (Fig. 4).

Additionally, astrocytes influence microglial activation and express CXCR3 in response to CNS inflammation [26]. As expected, with the low expression of CXCR3 at $48 \mathrm{~h}$ postIR, the inhibitory modulation of microglia by fluorocitrate in the injured spinal cord was simultaneously accompanied by astrocyte deactivation by minocycline in the same regions, which confirmed both the above results and the existence of CXCR3-mediated crosstalk between microglia and astrocytes in IR-induced pain (Fig. 4). Interestingly, in this study, although the intrathecal injections of minocycline and fluorocitrate exerted similar inhibitory effects on GFAP immunoreactivity at $12 \mathrm{~h}$ post-IR, the injection of minocycline decreased the levels of the CXCR3 protein, whereas the injection of fluorocitrate did not, suggesting that the early overexpression of CXCR3 in activated microglia was a major trigger of sequential astrocytic activation. Collectively, the results of this study provide evidence remonstrating that the interaction and crosstalk between microglia and astrocytes might be mediated by the chemokine-receptor pair CXCL10/CXCR3.

Because glial cells are the main effectors and regulators of the spinal cord, activated populations of glial cells could produce a wide variety of proinflammatory cytokines that strongly affect the pathogenesis of sensory hypersensitivity [6, 8, 17, 22]. Among these cytokines, TNF- $\alpha$ and IL- 6 are known to function as potent proinflammatory cytokines during the reperfusion period $[6,27,28]$. Consistent with our observation of bimodal allodynia induced during IR injury, two periods of robust TNF- $\alpha$ production were previously observed in an in vivo study [6]. An immediate peak in TNF- $\alpha$ production was detected at $12 \mathrm{~h}$, and a delayed peak was observed at $48 \mathrm{~h}$ after IR [6]. These data provide a plausible explanation for the phenomenon that the PWTs and PWLs decreased again after $12 \mathrm{~h}$. Moreover, as indicated by our ELISA results, the biphasic increases in TNF- $\alpha$ and IL- 6 levels were prevented by the intrathecal injection of AMD487 and minocycline, which eliminated the peak at $12 \mathrm{~h}$ post-IR, and by AMD487, minocycline and fluorocitrate, which eliminated the peak at $48 \mathrm{~h}$ (Fig. 5). These results were completely consistent with the contributions of differentially overexpressed CXCR3 to microglia and astrocyte activation during bimodal pain processing.

Notably, in addition to being expressed in microglia and astrocytes, CXCR3 was also found to be expressed in neurons of the periaqueductal gray matter in a recent study of the 


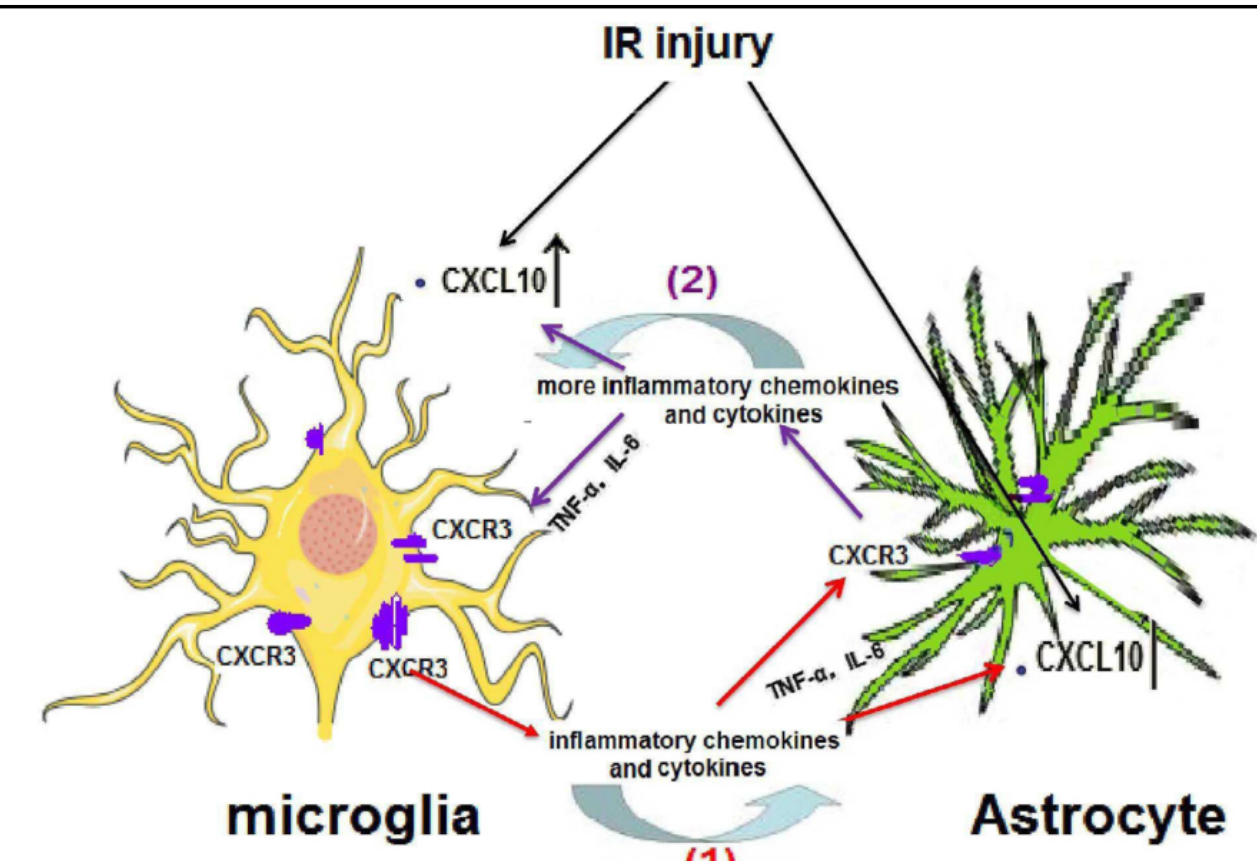

(1)

Fig. 6. Schematic showing the roles of the chemokine-receptor pair CXCL10/CXCR3 in the sequential activation of and crosstalk between spinal microglia and astrocytes in IR-induced bimodal inflammatory pain. IR injury induced bimodal inflammatory allodynia. During the early stage, IR-induced increases in CXCL10 expression first stimulated microglial activation and the release of proinflammatory cytokines (e.g., TNF-alpha and IL-6) by inducing the overexpression of CXCR3. These effects increased the expression of CXCL10 and CXCR3 on both microglia and astrocytes and ultimately amplified the inflammatory cascade and pain processing during the late stage. (1) Early stage of bimodal allodynia, (2) late stage of bimodal allodynia.

analgesic effects of morphine, and these findings suggest a potential role for CXCL10/CXCR3 in the mechanism of morphine tolerance that involves the regulation of neuron-microglia interactions [19]. Furthermore, another study used a transgenic approach to examine the effects of chronic cerebral CXCL10 expression on the infiltration of leukocytes into the CNS and found that astrocytes represent a significant cellular source of CXCL10 [29]. The astrocyte-directed production of CXCR3, however, had no effect on microglial activation. In contrast to our findings, only CXCL10-positive neurons, but not CXCL10-positive microglia or astrocytes, were observed to be involved in hippocampal formation in both CXCR3 knockout and wild-type mice with lesions in the entorhinal cortex [22]. However, the phased appearance of chemokines in acute and chronic inflammation is clearly important [30]. These discrepancies in the results might be due to differences in the observed time points and models used in the various experimental protocols. These discrepancies were consistent with the results of experiments that have revealed contradictory roles for CXCL10 in cardiovascular diseases, such as beneficial effects on arteriogenesis but detrimental effects on atherosclerotic plaque and aneurysm formation [31].

Taken together, the results of our study provide evidence showing that bimodal inflammatory allodynia was initiated in the spinal cord after IR injury. During the early stage, the IR-induced increases in CXCL10 expression stimulated microglia activation and the release of proinflammatory cytokines (e.g., TNF- $\alpha$ and IL-6) through the overexpression of CXCR3, which in turn increased the expression of CXCL10 and CXCR3 on both microglia and astrocytes, ultimately amplifying the inflammatory cascade and pain processing during the late stage (Fig. 6). Thus, activated microglia might be a trigger in the early phase that 


\section{Cellular Physiology Cell Physiol Biochem 2018;49:2214-2228

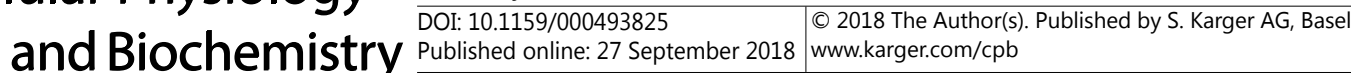 \\ Yu et al.: Sequential Glial Activation via CXCL10/CXCR3 Pair}

couples with astrocyte activation in the late phase to promote the development of IR-induced bimodal inflammatory pain via the chemokine-receptor pair CXCL10/CXCR3.

\section{Conclusion}

This study is the first to identify the involvement of the chemokine-receptor pair CXCL10/ CXCR3 in the development of bimodal inflammatory pain during the early reperfusion phase in a rat IR-induced pain model. The sequential activation of microglia and astrocytes and their crosstalk were mediated by CXCR3 overexpression, suggesting that treatments involving agents that act on specific cellular targets will be highly important in ameliorating IR-induced pain.

\section{Abbreviations}

ANOVA (one-way analysis of variance); BSCB (blood-spinal cord barrier); CNS (central nervous system); CXCL10 (C-X-C motif ligand 10); CXCR (C-X-C motif receptor); GFAP (glial fibrillary acidic protein); Iba-1 (ionized calcium-binding adaptor molecule-1); IFN (interferon); IL (interleukin); IR (ischemia-reperfusion); NF (nuclear factor); PWL (paw withdrawal latency); PWT (paw withdrawal threshold); rCXCL10 (recombinant rat CXCL10 protein); SEM (standard error of the mean); TNF (tumor necrosis factor).

\section{Disclosure Statement}

All authors declare no conflict of interest.

\section{References}

1 Li XQ, Zhang ZL, Tan WF, Sun XJ, Ma H: Down-Regulation of CXCL12/CXCR4 Expression Alleviates IschemiaReperfusion-Induced Inflammatory Pain via Inhibiting Glial TLR4 Activation in the Spinal Cord. PLoS One 2016;11:e0163807.

- Kwak KH, Jung H, Park JM, Yeo JS, Kim H, Lee HC, Byun SH, Kim JC, Park SS, Lim DG: A peroxynitrite decomposition catalyst prevents mechanical allodynia and NMDA receptor activation in the hind-paw ischemia reperfusion injury rats. Exp Ther Med 2014;7:508-512.

- Luo X, Tai WL, Sun L, Pan Z, Xia Z, Chung SK, Cheung CW: Crosstalk between astrocytic CXCL12 and microglial CXCR4 contributes to the development of neuropathic pain. Mol Pain DOI: $10.1177 / 1744806916636385$.

4 Saghaei E, Abbaszadeh F, Naseri K, Ghorbanpoor S, Afhami M, Haeri A, Rahimi F, Jorjani M: Estradiol attenuates spinal cord injury-induced pain by suppressing microglial activation in thalamic VPL nuclei of rats. Neurosci Res 2013;75:316-323.

-5 Fu Q, Zou MM, Zhu JW, Zhang Y, Chen WJ, Cheng M, Liu CF, Ma QH, Xu RX: TRIM32 affects the recovery of motor function following spinal cord injury through regulating proliferation of glia. Oncotarget 2017;8:45380-45390.

6 Smith PD, Puskas F, Meng X, Lee JH, Cleveland JC Jr, Weyant MJ, Fullerton DA, Reece TB: The evolution of chemokine release supports a bimodal mechanism of spinal cord ischemia and reperfusion injury. Circulation 2012;126:S110-117.

7 Karve IP, Taylor JM, Crack PJ: The contribution of astrocytes and microglia to traumatic brain injury. Br J Pharmacol 2016;173:692-702.

8 Zhang ZJ, Jiang BC, Gao YJ: Chemokines in neuron-glial cell interaction and pathogenesis of neuropathic pain. Cell Mol Life Sci 2017;74:3275-3291. 


\section{Cellular Physiology Cell Physiol Biochem 2018;49:2214-2228

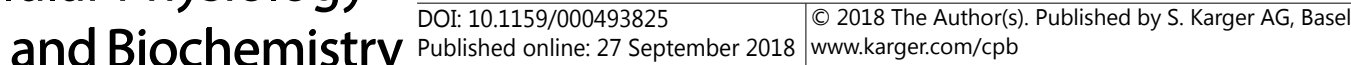 \\ Yu et al.: Sequential Glial Activation via CXCL10/CXCR3 Pair}

-9 Ding Q Quah SY, Tan KS: Secreted adenosine triphosphate from Aggregatibacter actinomycetemcomitans triggers chemokine response. Mol Oral Microbiol 2016;31:423-434.

10 Lee JH, Kim B, Jin WJ, Kim HH, Ha H, Lee ZH: Pathogenic roles of CXCL10 signaling through CXCR3 and TLR4 in macrophages and T cells: relevance for arthritis. Arthritis Res Ther 2017;19:163.

11 Krauthausen M, Saxe S, Zimmermann J, Emrich M, Heneka MT, Müller M: CXCR3 modulates glial accumulation and activation in cuprizone-induced demyelination of the central nervous system. J Neuroinflammation 2014;11:109.

12 Jiang BC, He LN, Wu XB, Shi H, Zhang WW, Zhang ZJ, Cao DL, Li CH, Gu J, Gao YJ: Promoted Interaction of C/EBP $\alpha$ with Demethylated Cxcr3 Gene Promoter Contributes to Neuropathic Pain in Mice. J Neurosci 2017;37:685-700.

13 Mcllwrath SL, Nesemeier R, Ma F, Oz HS, Zhang L, Westlund KN: Inflammatory 'double hit' model of temporomandibular joint disorder with elevated CCL2, CXCL9, CXCL10, RANTES and behavioural hypersensitivity in TNFR1/R2-/- mice. Eur J Pain 2017;21:1209-1223.

14 Guan XH, Fu QC, Shi D, Bu HL, Song ZP, Xiong BR, Shu B, Xiang HB, Xu B, Manyande A, Cao F, Tian YK: Activation of spinal chemokine receptor CXCR3 mediates bone cancer pain through an Akt-ERK crosstalk pathway in rats. Exp Neurol 2015;263:39-49.

15 van den Borne P, Haverslag RT, Brandt MM, Cheng C, Duckers HJ, Quax PH, Hoefer IE, Pasterkamp G, de Kleijn DP: Absence of chemokine (C-x-C motif) ligand 10 diminishes perfusion recovery after local arterial occlusion in mice. Arterioscler Thromb Vasc Biol 2014;34:594-602.

16 Li J, Ge M, Lu S, Shi J, Li X, Wang M, Huang J, Shao Y, Huang Z, Zhang J, Nie N, Zheng Y: Pro-inflammatory effects of the Th1 chemokine CXCL10 in acquired aplastic anaemia. Cytokine 2017;94:45-51.

17 Hernangómez M, Klusáková I, Joukal M, Hradilová-Svíženská I, Guaza C, Dubový P: CD200R1 agonist attenuates glial activation, inflammatory reactions, and hypersensitivity immediately after its intrathecal application in a rat neuropathic pain model. J Neuroinflammation 2016;13:43.

-18 Yang JL, Xu B, Li SS, Zhang WS, Xu H, Deng XM, Zhang YQ: Gabapentin reduces CX3CL1 signaling and blocks spinal microglial activation in monoarthritic rats. Mol Brain 2012;5:18.

19 Wang W, Peng Y, Yang H, Bu H, Guo G, Liu D, Shu B, Tian X, Luo A, Zhang X, Gao F: Potential role of CXCL10/ CXCR3 signaling in the development of morphine tolerance in periaqueductal gray. Neuropeptides 2017;65:120-127.

20 Duruisseaux M, Rabbe N, Antoine M, Vieira T, Poulot V, Cadranel J, Wislez M: Pro-tumoural CXCL10/CXCR3-A autocrine loop in invasive mucinous lung adenocarcinoma. ERJ Open Res DOI: 10.1183/23120541.00047-2016.

21 Lepej SZ, Rode OD, Jeren T, Vince A, Remenar A, Barsić B: Increased expression of CXCR3 and CCR5 on memory CD4+ T-cells migrating into the cerebrospinal fluid of patients with neuroborreliosis: the role of CXCL10 and CXCL11. J Neuroimmunol 2005;163:128-134.

22 Rappert A, Bechmann I, Pivneva T, Mahlo J, Biber K, Nolte C, Kovac AD, Gerard C, Boddeke HW, Nitsch R, Kettenmann H: CXCR3-dependent microglial recruitment is essential for dendrite loss after brain lesion. J Neurosci 2004;24:8500-8509.

-23 van Weering HR, Boddeke HW, Vinet J, Brouwer N, de Haas AH, van Rooijen N, Thomsen AR, Biber KP: CXCL10/CXCR3 signaling in glia cells differentially affects NMDA-induced cell death in CA and DG neurons of the mouse hippocampus. Hippocampus 2011;21:220-232.

24 Ha Y, Liu H, Xu Z, Yokota H, Narayanan SP, Lemtalsi T, Smith SB, Caldwell RW, Caldwell RB, Zhang W: Endoplasmic reticulum stress-regulated CXCR3 pathway mediates inflammation and neuronal injury in acute glaucoma. Cell Death Dis: 2015;6:e1900.

25 Matsumoto S, Matsumoto M, Yamashita A, Ohtake K, Ishida K, Morimoto Y, Sakabe T: The temporal profile of the reaction of microglia, astrocytes, and macrophages in the delayed onset paraplegia after transient spinal cord ischemia in rabbits. Anesth Analg 2003;96:1777-1784.

-26 Biber K, Dijkstra I, Trebst C, De Groot CJ, Ransohoff RM, Boddeke HW: Functional expression of CXCR3 in cultured mouse and human astrocytes and microglia. Neuroscience 2002;112:487-497.

27 Zhang J, Deng X: Bupivacaine effectively relieves inflammation-induced pain by suppressing activation of the NF- $\kappa B$ signalling pathway and inhibiting the activation of spinal microglia and astrocytes. Exp Ther Med 2017; 13:1074-1080.

28 Zhu P, Li JX, Fujino M, Zhuang J, Li XK: Development and treatments of inflammatory cells and cytokines in spinal cord ischemia-reperfusion injury. Mediators Inflamm 2013;2013:701970. 


\section{Cellular Physiology Cell Physiol Biochem 2018;49:2214-2228}

and Biochemistry \begin{tabular}{l|l} 
DOI: 10.1159/000493825 \\
Published online:27 September 2018
\end{tabular} $\begin{aligned} & \text { O } 2018 \text { The Author(s). Published by S. Karger AG, Basel } \\
& \text { www.karger.com/cpb }\end{aligned}$

Yu et al.: Sequential Glial Activation via CXCL10/CXCR3 Pair

29 Boztug K, Carson MJ, Pham-Mitchell N, Asensio VC, DeMartino J, Campbell IL: Leukocyte infiltration, but not neurodegeneration, in the CNS of transgenic mice with astrocyte production of the CXC chemokine ligand 10. J Immunol 2002;169:1505-1515.

30 Flynn G, Maru S, Loughlin J, Romero IA, Male D: Regulation of chemokine receptor expression in human microglia and astrocytes. J Neuroimmunol 2003;136:84-93.

-31 van den Borne P1, Haverslag RT, Brandt MM, Cheng C, Duckers HJ, Quax PH, Hoefer IE, Pasterkamp G, de Kleijn DP:Absence of chemokine ( $\mathrm{C}-\mathrm{x}-\mathrm{C}$ motif) ligand 10 diminishes perfusion recovery after local arterial occlusion in mice. Arterioscler Thromb Vasc Biol 2014;34:594-602. 